\title{
Modelling and analysis of river networks based on complex networks theory
}

\author{
Xuewen $\mathrm{Wu}$, Ling Li, Yonggang Qu \\ College of Computer and Information \\ Hohai University \\ Nanjing 210098, Jiangsu, China \\ hhuwxw@hhu.edu.cn, liling123156@sina.com, quyonggang1988@163.com
}

\begin{abstract}
River systems are open and self-organizing complex systems. Complex networks theory can well combine rivers' macro properties with their microscopic properties. This paper builds a river network model based on complex networks theory and describes its characteristics. After the analysis of the model used in Haihe River Basin, it shows that Haihe River Basin network has the small-world characteristics. This work provides a new approach to research the properties of river networks, so that to predict and control its behavior.
\end{abstract} Haihe

Key words-river network; complex networks; modelling;

\section{INTRODUCTION}

With certain hydraulic connections, A large number of rivers, intersections, and water conservancy facilities together constitute a criss-cross river networks system, which is far from equilibrium, open and self-organization.

If the river basin including rivers and water conservancy facilities is abstracted into a model of complex networks, all of the studies can be done on the network[1-4]. This paper will build a river network model based on the complex networks, study the statistical properties of the river network, and analyze the instance of Haihe basin. This is a new attempt, which maybe provides a theoretical basis or inspiration to professional technology research.

\section{CONSTRUCTION OF RIVER NETWORK MODEL}

\section{A. Node}

Positions with significant hydraulic characteristics are defined as nodes, including river sources, confluences of

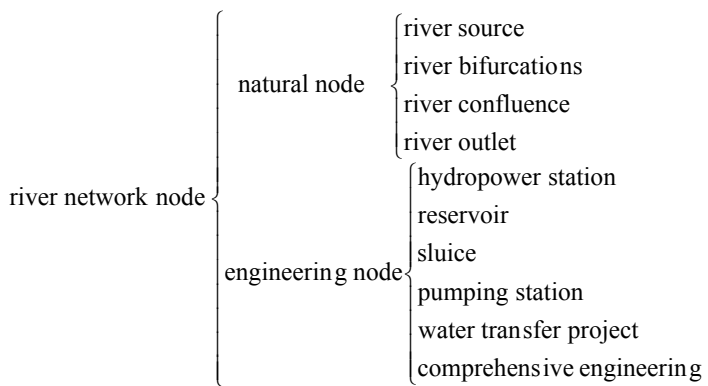

Figure 1. Node types rivers, water pumps and so on. They are divided into two categories in terms of hydraulic function: natural nodes and engineering nodes, as shown in Fig.1. Different methods are taken in different nodes.

Natural nodes include river sources, river confluences, river bifurcations, river outlets and so forth. These nodes do not contain any water conservancy facilities, which only have the characteristics of natural laws of the river itself.

Engineering nodes include hydropower stations, reservoirs, and sluices, pumping stations, water transfer projects, comprehensive engineering and other water conservancy project facilities as shown in Fig. 1. These water conservancy facilities play an important role in regulation and distribution of water resources, which can change the river flow under manual control and result in the significant different characteristics between upstream and downstream. Their hydraulic characteristics are more complex than that of natural nodes, which need to consider a variety of natural factors and human control factors. If a node is consistent with the definition of natural node and engineering node, then it is defined as an engineering node. For example, the water conservancy project built on junction of rivers is defined as an engineering node.

The set $V$ stands for the set of nodes, and $v_{i}=1,2$ stands for the type of nodes, which could distinguish between natural nodes and engineering nodes.

$$
\begin{gathered}
V=\left\{v_{1}, v_{2}, \cdots, v_{n}\right\} \\
v_{i}=\left\{\begin{array}{l}
1 ; \text { The node } i \text { is natural node } \\
2 ; \text { The node } i \text { is engineering node }
\end{array}\right.
\end{gathered}
$$

where $n$ is the total number of nodes in the river network.

\section{B. Edge}

The routes of rivers are defined as the edges of the river network. An edge is the link between two different nodes, including natural rivers and artificial channels. The direction of an edge is in accordance with that of the flow of the river. There are three basic types of links, which are series connection, parallel connection and mixed connection (both series connection and parallel connection).

Suppose that there are no isolate nodes or loopbacks in the network, and there is no more than one edge between two different nodes. 
$E$ is the set of edges. It is in the form

$$
E=\left\{\left\langle v_{i}, v_{j}\right\rangle \mid v_{i}, v_{j} \in V\right\}
$$

where $V$ is the set of nodes, and $v_{i}, v_{j}$ is the nodes, and $\left\langle v_{i}, v_{j}\right\rangle$ stands for the directed edge from the node $v_{i}$ to $v_{j}$.

Adjacency matrixes are utilized to represent the direction of edges [6-7]. A complex river network containing $n$ nodes can be mapped as an $n \times n$ adjacency matrix $R$ as

$$
R=\left[\begin{array}{cccccc}
r_{11} & r_{12} & \cdots & r_{1 j} & \cdots & r_{1 n} \\
r_{21} & r_{22} & \cdots & r_{2 j} & \cdots & r_{2 n} \\
\vdots & \vdots & \ddots & \vdots & \ddots & \vdots \\
r_{i 1} & r_{i 2} & \cdots & r_{i j} & \cdots & r_{i n} \\
\vdots & \vdots & \ddots & \vdots & \ddots & \vdots \\
r_{n 1} & r_{n 2} & \cdots & r_{n j} & \cdots & r_{n n}
\end{array}\right]
$$

where $r_{i j}$ stands for the type of the link from the node $v_{i}$ to $v_{j}$.

If $r_{i j}=1, r_{j i}=0$, it means there is a directed link from the node $v_{i}$ to $v_{j}$.

If $r_{i j}=r_{j i}=1$, it means that the link is two-way connection. On one hand, $v_{i}$ points to $v_{j}$, on the other hand, $v_{j}$ points to $v_{i}$. As the flow of rivers goes in only one direction without consideration of countercurrent phenomenon, the two-way connection does not exist in the river network.

If $r_{i j}=r_{j i}=0$, it means there is no link between nodes $v_{i}$ and $v_{j}$.

\section{Weight}

As different rivers may have different length and flow, the length and the flow are defined as the weights of the edges in complex river network.

$W$ is the set of weights in the form

$$
\left.W=\left\{w_{i j} \mid w_{i j}=\left(l_{i j}, x_{i j}^{i}(t)\right), x_{i j}^{o}(t)\right), v_{i}, v_{j} \in V\right\}
$$

where $l_{i j}$ is the length of the river. $x_{i j}^{i}(t)$ and $x_{i j}^{o}(t)$ are the flow rates of the upstream and the downstream of the river $r_{i j}$ varying with time. They are functions of time $t$.If $r_{i j}=1$ which means that there exists a riverway from the nodes $v_{i}$ to $v_{j}$, then $l_{i j}$ is defined as the real length of the river. Otherwise $r_{i j}$ is zero. If $r_{i j}=1$, then $x_{i j}^{i}(t)$ and $x_{i j}^{o}(t)$ are the actual flow rates of the upstream and

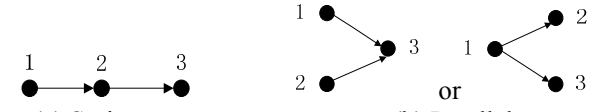

(a) Series

(b) Parallel

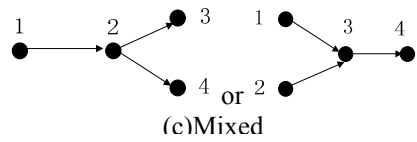

Figure 2. Three basic types of links Series

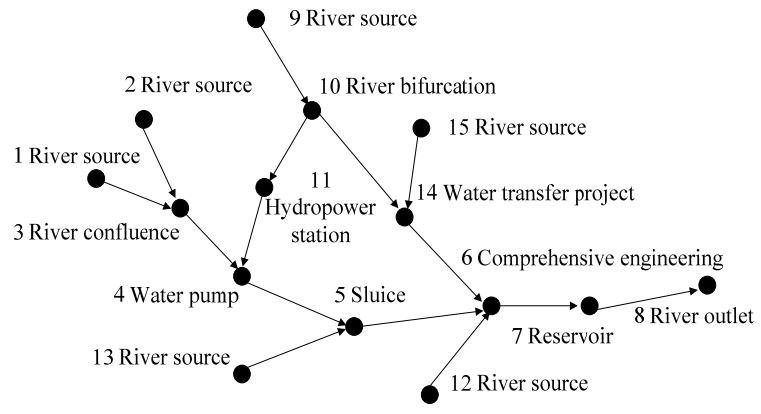

Figure 3. The topology of a typical river network

downstream. However, $x_{i j}^{i}(t)$ and $x_{i j}^{o}(t)$ are both equal to zero when $r_{i j}=0$.

\section{Topology}

There are three basic types of link between nodes that are series connection, parallel connection and mixed connection as shown in Fig. 2. Fig. 3 is the topology of a river network containing 15 nodes including the three basic categories of links with a certain degree of representativeness.

\section{E. Modelling of River Network}

By the above analysis, the river network can be abstracted as a directed complex networks model $(G)$. This model contains three basic elements, including a set of nodes $(V)$, a set of edges ( $E)$, and a set of weights $(W)$.

$$
G=\{V, E, W\}
$$

where $V=\left\{v_{1}, v_{2}, \cdots, v_{n}\right\}$ stands for the river network which contains $n$ nodes. $E=\left\{\left\langle v_{i}, v_{j}\right\rangle \mid v_{i}, v_{j} \in V\right\}$ is the set of edges. $\left.W=\left\{w_{i j} \mid w_{i j}=\left(l_{i j}, x_{i j}^{i}(t)\right), x_{i j}^{o}(t)\right), v_{i}, v_{j} \in V\right\}$ is the set of weights.

\section{THE DEGREE OF RIVER NETWORK}

\section{A. The Distribution of Degree}

Degree of a node is defined as the number of edges between the node with other nodes, which is divided into out-degree and in-degree. Out-degree refers to the number of edges the node points out to other nodes, which means the number of river channels that the river node flows to other 
river nodes in the complex river network. And in-degree is the number of edges other nodes point into this node, which shall be the number of river channels that other river nodes flow to this node. Degree is equal to the sum of out-degree and in-degree.

The average value of degree of all nodes is defined as the average degree of the network, marked as $\langle k\rangle$. The function $P(k)$ can be used to describe the distribution of degree in the network. $P(k)$ indicates the probability that the degree of a randomly selected node exactly is $k$, which is also equal to the ratio of the number of nodes whose degree is $k$ in to the total number of river network nodes.

The adjacency matrix can be used to describe degree. For a directed network, degree can be defined as

$$
\begin{aligned}
& k_{i}=k_{i}^{-}+k_{i}^{+} \\
& k_{i}^{-}=\sum_{j \in N} r_{i j} \\
& k_{i}^{+}=\sum_{j \in N} r_{j i}
\end{aligned}
$$

where $k_{i}^{-}$is out-degree and $k_{i}^{+}$is in-degree. $k_{i}$ is the degree of node $i$ and $r_{i j}$ is the connection relationship from node $i$ to node $j$.

The average degree of the network can be defined as

$$
<k>=\frac{1}{n} \sum_{i \in N} k_{i}
$$

where $\langle k\rangle$ is the average degree of the network, $k_{i}$ is the degree of node $i, n$ is the total number of nodes in the river network.

The degree of a node is the easiest but also the most important concept to depict the characteristics of a node. The degree can characterize the importance of a node in the network to a certain extent. For the river network, the greater the degree is, the more rivers intersect or bifurcate at the node, which means the more "importance" of the node in some sense and the more we need to adjust control and reallocate the water source.

\section{APPLICATION}

\section{A. Haihe River Basin Network Model}

Haihe River Basin is located in northern China, composed of seven main rivers, North Canal, Yongding River and Zhangweinan Canal and so on. In addition to Jiyun Canal, the rest of the rivers flow into Haihe River and finally Haihe River flows into Bohai Sea through Tianjin City. The length of Haihe River is $73 \mathrm{~km}$. Nowadays, many large-scale water conservancy constructions, including reservoirs, hydropower station and water conservancy, have been built in Haihe River Basin.

Reviewing the book of The Achievements of the Hydropower Resources of the People's Republic of China and other materials [7], we construct Haihe River Basin network model based on complex networks theory. The water system graph of Haihe River Basin is abstracted into a 565-node network graph, including 319 natural nodes and 246 engineering nodes. River sources, river confluences, river bifurcations, river outlets and so forth are defined as natural nodes, while the Miyun Reservoir, the Marco Polo Bridge hub and other engineering projects are defined as engineering nodes. North Canal, Yongding River, Zhangweinan Canal and other rivers or canals are abstracted into the edges of the network which contains 616 edges. Pajek implement is utilized to draw the network diagram of Haihe River Basin as shown in Fig.4.

\section{B. Data Analysis}

Different degrees of nodes generally have different characteristics in actual river network. The degree may reflect the type of a node and the property corresponding to the actual object.

The degree distribution of nodes in Haihe River Basin network model is calculated and obtained by using Matlab implement, as shown in Table 1.

From the degree distribution table (Table 1) and network diagram of Haihe River Basin (Fig. 4), we can get conclusions as follows:

$k=1$ is corresponding to natural nodes, which are always the sources of the entire river network.

$k=2$ is corresponding to engineering nodes, the majority of which are reservoirs, sluices and other hydraulic engineering facilities. These nodes always play an important role in usage and regulation of water resources.

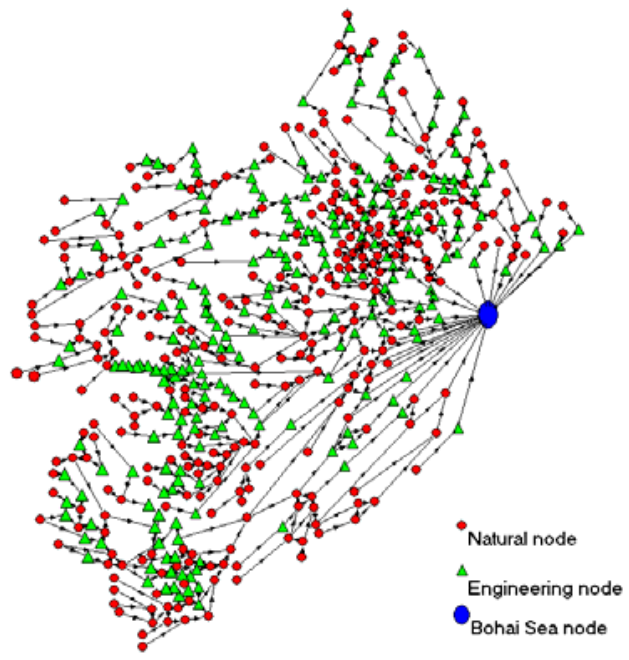

Figure 4. Network diagram of Haihe River Basin

TABLE I. DEGREE DistribUtion TABLE OF NODES IN HAIHE RIVER BASIN NETWORK MODEL

\begin{tabular}{c|ccccccccc}
\hline $\begin{array}{c}\text { The degree } \\
\text { of a node }\end{array}$ & 1 & 2 & 3 & 4 & 5 & 6 & 7 & $8 \sim 22$ & 23 \\
\hline Numeric & 156 & 219 & 151 & 32 & 3 & 2 & 1 & 0 & 1 \\
\hline
\end{tabular}


$k=3$ is maybe corresponding to natural nodes or engineering nodes, which are always river confluences or bifurcations. These nodes reallocate of water resources.

$k=4,5,6$ or 7 is mostly corresponding to engineering nodes where always some rivers converge or some hydraulic engineering facilities are built. These nodes play a pivotal hub part in the whole basin. For example, Huangbizhuang Reservoir is a large-scale water conservancy project, which mainly controls the floods and takes into account the comprehensive utilization of water usage, irrigation and power generation. The node where Huangbizhuang Reservoir is built is an engineering node with degree equal to 5. In August 1996, when the devastating flood came into the central and southern regions of Hebei Province, Huangbizhuang Reservoir played an very important role in winning time for downstream flood prevention and emergency rescue.

$k=23$ is corresponding to a natural node which is the only one node whose degree is 23 in Haihe River Basin network. It is Bohai Sea, which is river outlet and also the terminal point of the entire network.

$k=8 \sim 22$ does not exist in the river network, which explains that there is no other node whose degree is larger than most nodes in addition to Bohai Sea node. Analysing of the reason, we can get that there cannot be many rivers converging at one place due to the limitations of the twodimensional geographic plane. So the degree of nodes in the river network cannot be too large, which also reflects that the characteristics of river network are uniquely different from that of other networks.

According to degree distribution of nodes in Haihe River Basin network(Table 1), Plot the probability distribution figure of the degree as shown in Fig. 5.

From Table 1 and Fig. 5, we can get that there are 526 nodes whose degree is 1,2 , or 3 , which account for $93.09 \%$

of the total number of the network nodes. There are only 38 nodes whose degree is $4,5,6$, or 7 , accounting for $6.73 \%$, while only one node's degree is 23 accounting for $0.18 \%$. It reflects that there are many nodes which have a few numbers of links with other nodes. A small amount of nodes have a moderate number of links, while only a very few nodes have large number of links.

The degree about $66.37 \%$ of nodes is larger than the

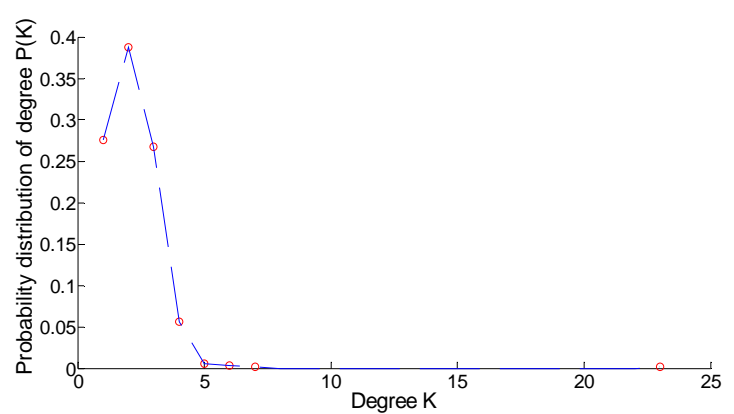

Figure 5. The probability distribution figure of the degree of nodes in Haihe River Basin network average degree. They play an auxiliary connecting role, while the degree about $33.63 \%$ of the nodes is smaller and these nodes play a pivotal hub part in the whole network.

As shown in Table 1, there is no node with degree equal to 0 , which means that it does not exit an isolated node. So the entire river network is interconnected. The majority of the nodes are only connected to their adjacent nodes. The distribution curve of degree has a peak near the mean value of degree and is attenuated speedily away from the peak. So Haihe River Basin network has the characteristics of smallworld networks to some extent.

\section{CONCLUSION}

In this paper, we abstract and conceptualize the river basin, generate network nodes diagram, build complex river network model and further analyze the statistical properties of the degree distribution. After the analysis of the model used in Haihe River Basin, Haihe River Basin network has the small world properties in a certain extent.

Complex networks can be used to systematically describe the river network, which provides a new idea for the study of the complex character of river network and the planning of river water resources. With the application of the model, we can further study the flow characteristics and hydrological characteristics of river network, research its operation mode and then predict and control the behavior of the river network system.

\section{REFERENCES}

[1] I. G. Pechlivanidis, B. M. Jackson, N. R. Mcintyre and H. S. Wheater, "Catchment scale hydrological modeling: a review of model types, calibration approaches and uncertainty analysis methods in the context of recent developments in technology and applications," Global NEST Journal, Vol.13, pp.193-214, 2011.

[2] I. Rodriguez-Iturbe, A. Rinaldo, R. Rigon, R.L. Bras, E. IjjaszVasquez and A. Marani, "Fractal structures as least energy dissipation patterns: the case of river networks," Geophysical Research Letters, Vol.5, pp. 2854-2860, 1992.

[3] P. S. Dodds and D. H. Rothman, "Geometry of river networks: Scaling, fluctuations and deviations," Physical Review E, vol.63, pp. 193-214, 2000.

[4] Yongchen Zong, "The research of nonlinear characteristics and fractal in the river-network," Doctor, Tianjing: Tianjin University, 2007.

[5] Daren He, Zonghua Liu and Binghong Wang, "Complex Systems and Complex Networks," Beijing: Higher Education Press, 2009.

[6] Xiaofan Wang, Xiang Li and Guanrong Chen, "Complex Networks Theory and its Application," Beijing: Tsinghua University Press, 2006.

[7] "Review the achievements of the hydropower resources of the People's Republic of China (2003), vol.Beijing, Tianjin and Hebei Province," Beijing: China Electric Power Press, 2004. 